\title{
Regularity for MHD Second Grade Fluid in a Porous Space
}

\author{
S. Rahman, T. Hayat, and Hamed H. Alsulami
}

\begin{abstract}
The purpose of present paper is to establish the regularity of classical solution for second grade fluid. We consider unsteady flow passing through the porous medium. The incompressible fluid is taken between two rigid plates. We apply the magnetic field of the system and Darcy's law is performed due to porous medium. In addition uniqueness of classical solution is also verified. Finally neglecting the effect of porosity we obtain a unique classical solution of MHD second grade fluid.
\end{abstract}

Index Terms-Porous medium, second grade fluid, classical solution.

\section{INTRODUCTION}

Several materials (non-Newtonian fluids) in industrial and technological applications differ greatly from Newtonian fluids in their rheological characteristics. These liquids do not obey the Newton's law of viscosity. In particular, such fluids are encountered in geophysics, chemical and nuclear industries, material processing, petroleum and many others. Also all the non-Newtonian fluids cannot be described by one constitutive equation. Researchers in the field are using several constitutive relationships for the non-Newtonian fluids. Much attention in the past has been devoted to the flows of second grade fluid (a simplest subclass of differential type non-Newtonian fluids). Numerous studies have been made for the one dimensional unsteady flows of second grade fluid (see [1]-[10] for few latest articles). Motivated by such facts, our intention here is to develop regularity of unique classical solution for unsteady flow of MHD second grade fluid in a porous medium. The fluid is consider between two plates. We apply the magnetic field of the system and Darcy's law is performed due to porous medium.

\section{DESCRIPTION OF THE PROBLEM}

We consider the flow of a MHD second grade non-Newtonian fluid between two horizontal parallel rigid

Manuscript received November 5, 2013; revised January 16, 2014. This work was supported by the National Natural Science Foundation of China (Grant No. 11271299), the Mathematical Tiyanyuan Foundation of China (Grant No. 11126027) and Natural Science Foundation Research Project of Shaanxi Provience (2012JM1014). Further the research of Dr. Alsulami was partially supported by the Deanship of Scientific Research (DSR), King Abdulaziz University, Jeddah, Suaidi Arabia.

S. Rahman is with the Department of Applied Mathematics, Northwestern Polytechnical University, 710129, Xi'an, Shaanxi, P. R. China (e-mail: saeed@ ciit.net.pk).

T. Hayat is with the Department of Mathematics, Quaid-i-Azam University 45320 Islamabad 44000, Pakistan.

Hamed H. Alsulami is with the Nonlinear Analysis and Applied Mathematics (NAAM) Research Group, Faculty of Science, King Abdulaziz University, Jeddah 21589, Saudi Arabia. plates. The $\mathrm{x}$-axis is taken parallel to rigid plates $\mathrm{y}$-axis is perpendicular to $\mathrm{x}$-axis.

For the unidirectional flow, the velocity field is

$$
\vec{V}=(u(y, t), 0)
$$

where the above definition of velocity automatically satisfies the incompressibility condition. The equation of motion in a porous medium without body forces is

$$
\rho \frac{d \vec{V}}{d t}=\operatorname{div} \vec{\sigma}+\vec{r}
$$

where $\rho$ is the density of fluid, $\frac{d \vec{V}}{d t}$ is the material time differentiation, $\vec{\sigma}$ is the Cauchy stress tensor, and $\vec{r}$ is the Darcy's resistance in a porous space. The Cauchy stress tensor of an incompressible second grade fluid has the form [11]-[15]

$$
\vec{\sigma}=-p \vec{I}+\mu \vec{A}_{1}+\vec{\alpha}_{1} \vec{A}_{2}+\vec{\alpha}_{2} \vec{A}_{1}^{2}
$$

in which $p$ is the pressure, $\vec{I}$ is the identity tensor, $\alpha_{i}(i=1,2)$ are the normal stress moduli, and $\vec{A}_{i}(i=1,2)$ are the first two Rivlin-Ericksen is defined as

$$
\begin{gathered}
\vec{A}_{1}=(\operatorname{grad} \vec{V})+(\operatorname{grad} \vec{V})^{T}, \\
\vec{A}_{2}=\frac{d \vec{A}_{1}}{d t}+\vec{A}_{1}(\operatorname{grad} \vec{V})+(\operatorname{grad} \vec{V})^{T} \vec{A}_{1} .
\end{gathered}
$$

In studying fluid dynamics, If (3) is required to be compatible with thermodynamics, then the material moduli must meet the following restrictions

$$
\mu \geq 0, \quad \alpha_{1} \geq 0, \quad\left|\alpha_{1}+\alpha_{2}\right|=0 .
$$

For the unsteady flow in porous medium, the Darcy resistance for a second grade fluid, which is a measure of the flow resistance offered by the solid matrix, following Vafai and Tien [16], is given by

$$
\vec{r}_{x}=-\frac{\phi}{K}\left(\mu+\alpha_{1} \frac{\partial}{\partial t}\right) u .
$$

From (1) - (5) and (7) and for simplicity ignoring arrow for vector quantity, we have

$$
\rho \frac{\partial u}{\partial t}=\mu \frac{\partial^{2} u}{\partial y^{2}}+\alpha_{1} \frac{\partial^{3} u}{\partial y^{2} \partial t}-\frac{\phi}{K}\left(\mu+\alpha_{1} \frac{\partial}{\partial t}\right) u-\sigma B_{0}^{2} u
$$


The relevant initial conditions is

$$
u(y, 0)=u_{0}(y)
$$

where $u_{0}(y)$ is the initial velocity and $u$ vanishes at the lower and upper plates.

The literature on the Navier-Stokes is quite extensive but investigators of third grade fluid are few. The pioneer work for a global weak solution $u(x, t)$ of the Navier-Stokes equations belong to $L^{\infty}\left(0, T ; L^{2}\left(R^{3}\right)\right) \cap L^{2}\left(0, T ; H^{1}\left(R^{3}\right)\right)$ with $u_{0} \in L^{2}\left(R^{3}\right)$ and $d i v u_{0}=0$ was constructed by Leray [16] and Hopf [17], which is known as Leray -Hopf weak solution. Serrin [18] showed that if $u$ is Leray-Hopf weak solution in $L^{\alpha, \gamma}=L^{\alpha}\left(0, T ; L^{\gamma}\left(R^{3}\right)\right) \quad$ with $\frac{2}{\alpha}+\frac{3}{\gamma} \leq 1,2<\alpha<\infty, 2<\gamma<\infty, \quad$ then the solution $u(x, t) \in C^{\infty}\left(R^{3} \times(0, T)\right)$. The partial regularity theory of Navier-Stokes equations were proved by Scheffer [19] and further improvement was made by Caaffarelli et al. [20] and Tian and Xin in [21]. Further more in [22], Sohr introduced Lorentz spaces and proved $u \in L^{s, r}\left(0, T ; L^{q, \infty}\right)$ with $\frac{2}{s}+\frac{3}{q}=1,3<q<\infty, 2<s<r<\infty$, which validate the strong energy inequality.

Serrin regularity class of weak solution of the NavierStokes equations was further modified in Sohr [23] by introducing the Sobolev spaces of negative order, and derived

with $u \in L^{s}\left(0, T ; L^{-\alpha, q}\right) \frac{2}{s}+\frac{3}{q}=1-\alpha, 3<q<\infty, \quad 2<s<r<\infty$, for $0<\alpha<1$. If $u$ is a weak solution in $C\left((0, T) ; L^{3}\left(R^{3}\right)\right)$, then Wahl and Giga in [24] and [25] proved $u(x, t) \in C^{\infty}\left(R^{3} \times(0, T)\right)$. The same regularity of $u \in L^{\infty}\left(0, T ; L^{3}\left(R^{3}\right)\right)$, is proved in [26] by taking sup $\|\nabla u\|_{L^{2}}^{2}$ is sufficiently small. The regularity of weak $0 \leq t_{0} \leq t$

solution $u(x, t) \in C^{\infty}\left(R^{3} \times(0, T)\right)$ was obtained in Kozono and Shor [27] provided $u(x, t)$ is left continuous with respect to $L^{3}$ norm for $t \in(0, T)$.

Cao and $\mathrm{Wu}$ [28] used the idea that the maximum norm of the vorticity controls the breakdown of smooth solutions of the 2D MHD equations with mixed partial dissipation and magnetic diffusion. In other words, if a weak solution of the 2D MHD equations with mixed partial dissipation and magnetic diffusion is initially smooth and loss its regularity at some later time, then the maximum vorticity necessary grows with out bound as the critical time approaches. That is if the velocity field $u$ of a weak solution and Vorticity $w=\nabla \times u$ satisfies

$$
\int_{0}^{T}\|\nabla u\|_{\infty} d t=\int_{0}^{T}\|w\|_{\infty} d t<\infty
$$

Then this solution is a classical solution on $[0, T]$. We apply this idea [28] and find the classical solution of MHD second grade fluid in a porous medium.

Our main result is

\section{Theorem 1}

Assuming $u_{0} \in W_{2}^{2,1}$ then the equations (8) and (9) have a unique classical solution $u$ satisfying

$$
u \in L^{\infty}\left([0, T] ; W_{2}^{2,1}\left(Q_{T}\right)\right), u \in L^{\infty}\left([0, T] ; W_{2}^{2,1}\left(Q_{T}\right)\right)
$$

where $w=\nabla \times u=-\frac{\partial u}{\partial y} \quad$ represent the vorticity and $Q_{T}=R^{2+} \times[0, T]$

\section{PROOF OF THEOREM 1}

For proving Theorem 1, we need several propositions.

\section{A. Proposition 1}

If $u$ is a solution of Eqs (8) and (9) then vorticity $w=\nabla \times u=-\frac{\partial u}{\partial y}$ satisfies

$$
\begin{gathered}
\sup _{0 \leq t \leq T}\left(\left(\rho+\frac{\alpha_{1} \phi}{K}\right)\|u\|_{L^{2}}^{2}+\alpha_{1}\left\|\frac{\partial u}{\partial y}\right\|_{L^{2}}^{2}\right) \\
\leq C\left(\left(\rho+\frac{\alpha_{1} \phi}{K}\right)\left\|u_{0}\right\|_{L^{2}}^{2}+\alpha_{1}\left\|\frac{\partial u_{0}}{\partial y}\right\|_{L^{2}}^{2}\right), \\
\sup _{0 \leq t \leq T}\left(\left(\rho+\frac{\alpha_{1} \phi}{K}\right)\|w\|_{L^{2}}^{2}+\alpha_{1}\left\|\frac{\partial w}{\partial y}\right\|_{L^{2}}^{2}\right) \\
\leq C\left(\left(\rho+\frac{\alpha_{1} \phi}{K}\right)\left\|w_{0}\right\|_{L^{2}}^{2}+\alpha_{1}\left\|\frac{\partial w_{0}}{\partial y}\right\|_{L^{2}}^{2}\right),
\end{gathered}
$$

where $C$ depends on $T$

\section{B. Proof}

Taking the inner product of Eq (8) with $u$ and using integration by parts, we obtain

$$
\begin{gathered}
\frac{1}{2}\left(\rho+\frac{\alpha_{1} \phi}{K}\right) \int_{R^{2+}} \frac{\partial}{\partial t}|u|^{2} \partial y \partial x=-\mu \int_{R^{2+}}\left(\frac{\partial u}{\partial y}\right)^{2} \partial y \partial x \\
-\alpha_{1} \int_{R^{2+}} \frac{\partial u}{\partial y} \frac{\partial^{2} u}{\partial y \partial t} \partial y \partial x-\left(\frac{\mu \phi}{K}+\sigma B_{0}^{2}\right) \int_{R^{2+}}|u|^{2} \partial y \partial x \\
\frac{d}{d t}\left(\left(\rho+\frac{\alpha_{1} \phi}{K}\right)\|u\|_{L^{2}}^{2}+\alpha 1\left\|\frac{\partial u}{\partial y}\right\|_{L^{2}}^{2}\right)=-2 \mu\left\|\frac{\partial u}{\partial y}\right\|_{L^{2}}^{2} \\
-\left(\frac{2 \mu \phi}{K}+2 \sigma B_{0}^{2}\right)\|u\|_{L^{2}}^{2} \\
\leq\left(\frac{2 \mu \phi}{K}+2 \sigma B_{0}^{2}\right)\|u\|_{L^{2}}^{2}+2 \mu\left\|\frac{\partial u}{\partial y}\right\|_{L^{2}}^{2}
\end{gathered}
$$




$$
\leq\left(\rho+\frac{\alpha_{1} \phi}{K}\right)\|u\|_{L^{2}}^{2}+\alpha_{1}\left\|\frac{\partial u}{\partial y}\right\|_{L^{2}}^{2},
$$

where $\frac{2 \mu \phi}{K}+2 \sigma B_{0}^{2} \leq \rho+\frac{\alpha_{1} \phi}{K}$ and $2 \mu \leq \alpha_{1}$. Now using Gronwall's inequality, we have

$$
\begin{aligned}
& \sup _{0 \leq t \leq T}\left(\left(\rho+\frac{\alpha_{1} \phi}{K}\right)\|u\|_{L^{2}}^{2}+\alpha_{1}\left\|\frac{\partial u}{\partial y}\right\|_{L^{2}}^{2}\right) \\
& \leq C\left(\left(\rho+\frac{\alpha_{1} \phi}{K}\right)\left\|u_{0}\right\|_{L^{2}}^{2}+\alpha_{1}\left\|\frac{\partial u_{0}}{\partial y}\right\|_{L^{2}}^{2}\right),
\end{aligned}
$$

where $C$ depends on $T$.

From (8) and (9), the vorticity $w$ satisfies the following equations

$$
\begin{gathered}
\left(\rho+\frac{\alpha_{1} \phi}{K}\right) \frac{\partial w}{\partial t}=\mu \frac{\partial^{2} w}{\partial y^{2}}+\alpha_{1} \frac{\partial^{3} w}{\partial y^{2} \partial t}-\left(\frac{\phi \mu}{K}+\sigma B_{0}^{2}\right) w \\
w(y, 0)=w_{0}(y) .
\end{gathered}
$$

Taking inner product of (10) with $w$ and integrating by parts, we get

$$
\begin{gathered}
\frac{1}{2}\left(\rho+\frac{\alpha_{1} \phi}{K}\right) \frac{d}{d t}\|w\|_{L^{2}}^{2}=-\mu\left\|\frac{\partial w}{\partial y}\right\|_{L^{2}}^{2}+\frac{\alpha_{1}}{2} \frac{d}{d t}\left\|\frac{\partial w}{\partial y}\right\|_{L^{2}}^{2} \\
-\left(\frac{\phi \mu}{K}+\sigma B_{0}^{2}\right)\|w\|_{L^{2}}^{2} \\
\frac{d}{d t}\left(\left(\rho+\frac{\alpha_{1} \phi}{K}\right)\|w\|_{L^{2}}^{2}+\alpha_{1}\left\|\frac{\partial w}{\partial y}\right\|_{L^{2}}^{2}\right)=-2 \mu\left\|\frac{\partial w}{\partial y}\right\|_{L^{2}}^{2} \\
-\left(\frac{2 \phi \mu}{K}+2 \sigma B_{0}^{2}\right)\|w\|_{L^{2}}^{2} \\
\leq\left(\frac{2 \phi \mu}{K}+2 \sigma B_{0}^{2}\right)\|w\|_{L^{2}}^{2}+2 \mu\left\|\frac{\partial w}{\partial y}\right\|_{L^{2}}^{2} \\
\leq\left(\rho+\frac{\alpha_{1} \phi}{K}\right)\|w\|_{L^{2}}^{2}+\alpha_{1}\left\|\frac{\partial w}{\partial y}\right\|_{L^{2}}^{2}
\end{gathered}
$$

where $\frac{2 \phi \mu}{K}+2 \sigma B_{0}^{2} \leq \rho+\frac{\alpha_{1} \phi}{K}$ and $2 \mu \leq \alpha_{1}$. Now using Gronwall's inequality, we have

$$
\begin{aligned}
& \sup _{0 \leq t \leq T}\left(\left(\rho+\frac{\alpha_{1} \phi}{K}\right)\|w\|_{L^{2}}^{2}+\alpha_{1}\left\|\frac{\partial w}{\partial y}\right\|_{L^{2}}^{2}\right) \\
& \leq C\left(\left(\rho+\frac{\alpha_{1} \phi}{K}\right)\left\|w_{0}\right\|_{L^{2}}^{2}+\alpha_{1}\left\|\frac{\partial w_{0}}{\partial y}\right\|_{L^{2}}^{2}\right)
\end{aligned}
$$

where $C$ depends on $T$

\section{Proposition 2}

If $w$ is a solution of Eqs (10) and (11) then

$$
\begin{aligned}
& \sup _{0 \leq t \leq T}\left(\left(\rho+\frac{\alpha_{1} \phi}{K}\right)\left\|\frac{\partial w}{\partial y}\right\|_{L^{2}}^{2}+\alpha_{1}\left\|\frac{\partial^{2} w}{\partial y^{2}}\right\|_{L^{2}}^{2}\right) \\
& \leq C\left(\left(\rho+\frac{\alpha_{1} \phi}{K}\right)\left\|\frac{\partial w_{0}}{\partial y}\right\|_{L^{2}}^{2}+\alpha_{1}\left\|\frac{\partial^{2} w_{0}}{\partial y^{2}}\right\|_{L^{2}}^{2}\right),
\end{aligned}
$$

\section{$C$ depends on $T$}

\section{Proof}

Taking inner product of (10) with $-\frac{\partial^{2} w}{\partial y^{2}}$ and integrating by parts, we get

$$
\begin{gathered}
\frac{1}{2}\left(\rho+\frac{\alpha_{1} \phi}{K}\right) \int_{R^{2+}} \frac{\partial}{\partial t}\left|\frac{\partial w}{\partial y}\right|^{2} \partial y \partial x=-\mu \int_{R^{2+}}\left|\frac{\partial^{2} w}{\partial y^{2}}\right|^{2} \partial y \partial x \\
-\frac{\alpha_{1}}{2} \int_{R^{2+}} \frac{\partial}{\partial t}\left|\frac{\partial^{2} w}{\partial y^{2}}\right|^{2} \partial y \partial x-\left(\frac{\phi \mu}{K}+\sigma B_{0}^{2}\right) \int \frac{\partial w}{R^{2+}}\left|\frac{\partial y}{\partial y}\right|^{2} \partial x \\
\frac{d}{d t}\left(\left(\rho+\frac{\alpha_{1} \phi}{K}\right)\left\|\frac{\partial w}{\partial y}\right\|_{L^{2}}^{2}+\alpha_{1}\left\|\frac{\partial^{2} w}{\partial y^{2}}\right\|_{L^{2}}^{2}\right)=-2 \mu\left\|\frac{\partial w}{\partial y}\right\|_{L^{2}}^{2} \\
-\left(\frac{2 \phi \mu}{K}+2 \sigma B_{0}^{2}\right)\left\|\frac{\partial w}{\partial y}\right\|_{L^{2}}^{2} \\
\leq\left(\frac{2 \phi \mu}{K}+2 \sigma B_{0}^{2}\right)\|w\|_{L^{2}}^{2}+2 \mu\left\|\frac{\partial w}{\partial y}\right\|_{L^{2}}^{2} \\
\leq\left(\rho+\frac{\alpha_{1} \phi}{K}\right)\|w\|_{L^{2}}^{2}+\alpha_{1}\left\|\frac{\partial w}{\partial y}\right\|_{L^{2}}^{2}
\end{gathered}
$$

where $\frac{2 \phi \mu}{K}+2 \sigma B_{0}^{2} \leq \rho+\frac{\alpha_{1} \phi}{K}$ and $2 \mu \leq \alpha_{1}$. Now using Gronwall's inequality, we have

$$
\begin{aligned}
& \sup _{0 \leq t \leq T}\left(\left(\rho+\frac{\alpha_{1} \phi}{K}\right)\left\|\frac{\partial w}{\partial y}\right\|_{L^{2}}^{2}+\alpha_{1}\left\|\frac{\partial^{2} w}{\partial y^{2}}\right\|_{L^{2}}^{2}\right) \\
& \leq C\left(\left(\rho+\frac{\alpha_{1} \phi}{K}\right)\left\|\frac{\partial w_{0}}{\partial y}\right\|_{L^{2}}^{2}+\alpha_{1}\left\|\frac{\partial^{2} w_{0}}{\partial y^{2}}\right\|_{L^{2}}^{2}\right),
\end{aligned}
$$

where $C$ depends on $T$

\section{E. Proof of Theorem 1}

For elementary inequality (see Lemma 14 of [29])

$$
\|f\|_{\infty} \leq\|f\|_{2}+\|\nabla f\|_{2}+\|\Delta f\|_{2}, \quad f \in C^{2}
$$

We have by using $f=w$ and propositions 1 and 2 that

$$
\int_{0}^{t}\|w(\tau) d \tau\|_{\infty} d \tau<\infty,
$$

for $t \in[0, T]$.

For the uniqueness, we assume that $u_{1}$ and $u_{2}$ are two solutions of (8) having the same initial conditions. Therefore $\rho \frac{\partial u_{1}}{\partial t}=\mu \frac{\partial^{2} u_{1}}{\partial y^{2}}+\alpha_{1} \frac{\partial^{3} u_{1}}{\partial y^{2} \partial t}-\frac{\phi}{K}\left(\mu+\alpha_{1} \frac{\partial}{\partial t}\right) u_{1}-\sigma B_{0}^{2} u_{1}(12)$ 


$$
\rho \frac{\partial u_{2}}{\partial t}=\mu \frac{\partial^{2} u_{2}}{\partial y^{2}}+\alpha_{1} \frac{\partial^{3} u_{2}}{\partial y^{2} \partial t}-\frac{\phi}{K}\left(\mu+\alpha_{1} \frac{\partial}{\partial t}\right) u_{2}-\sigma B_{0}^{2} u_{2}
$$

Subtracting (13) from (12) and letting $u_{1}-u_{2}=g$, we have

$$
\begin{gathered}
\rho \frac{\partial g}{\partial t}=\mu \frac{\partial^{2} g}{\partial y^{2}}+\alpha_{1} \frac{\partial^{3} g}{\partial y^{2} \partial t}-\frac{\phi}{K}\left(\mu+\alpha_{1} \frac{\partial}{\partial t}\right) g-\sigma B_{0}^{2} g, \\
g(y, 0)=g_{0}(y) .
\end{gathered}
$$

Taking the inner product of (14) with $g$ and after using integration by parts, we have

$$
\begin{gathered}
\frac{1}{2}\left(\rho+\frac{\alpha_{1} \phi}{K}\right) \int_{R^{2+}} \frac{\partial}{\partial t}|g|^{2} \partial y \partial x=-\mu \int_{R^{2+}}\left(\frac{\partial g}{\partial y}\right)^{2} \partial y \partial x \\
-\alpha_{1} \int_{R^{2+}} \frac{\partial u}{\partial y} \frac{\partial^{2} g}{\partial y \partial t} \partial y \partial x-\left(\frac{\mu \phi}{K}+\sigma B_{0}^{2}\right) \int_{R^{2+}}|g|^{2} \partial y \partial x, \\
\frac{d}{d t}\left(\left(\rho+\frac{\alpha_{1} \phi}{K}\right)\|g\|_{L^{2}}^{2}+\alpha_{1}\left\|\frac{\partial g}{\partial y}\right\|_{L^{2}}^{2}\right) \\
\leq\left(\frac{2 \mu \phi}{K \rho}+2 \sigma B_{0}^{2}\right)\|g\|_{L^{2}}^{2}+2 \mu\left\|\frac{\partial g}{\partial y}\right\|_{L^{2}}^{2} \\
\leq\left(\rho+\frac{\alpha_{1} \phi}{K}\right)\|g\|_{L^{2}}^{2}+\alpha_{1}\left\|\frac{\partial g}{\partial y}\right\|_{L^{2}}^{2},
\end{gathered}
$$

where $\frac{2 \mu \phi}{K}+2 \sigma B_{0}^{2} \leq \rho+\frac{\alpha_{1} \phi}{K}$ and $2 \mu \leq \alpha_{1}$. Now using Gronwall's inequality, we have

$$
\left(\rho+\frac{\alpha_{1} \phi}{K}\right)\|g\|_{L^{2}}^{2}+\alpha_{1}\left\|\frac{\partial g}{\partial y}\right\|_{L^{2}}^{2} \leq 0
$$

which implies that $\|g\|_{L^{2}}=0$, that is $g=0$ and $u_{1}=u_{2}$. Theorem 1 is completed.

For the special case when we assume that the medium is not porous then we take $\phi$ in (8) and find some results.

\section{F. Corollary 1}

If $u$ is a solution of Eqs (8) and (9) with $\phi=0$ then velocity $u$ and vorticity $w=\nabla \times u=-\frac{\partial u}{\partial y}$ satisfies

$$
\begin{aligned}
& \sup _{0 \leq t \leq T}\left(\rho\|u\|_{L^{2}}^{2}+\alpha_{1}\left\|\frac{\partial u}{\partial y}\right\|_{L^{2}}^{2}\right) \\
& \leq C\left(\rho\left\|u_{0}\right\|_{L^{2}}^{2}+\alpha_{1}\left\|\frac{\partial u_{0}}{\partial y}\right\|_{L^{2}}^{2}\right), \\
& \sup _{0 \leq t \leq T}\left(\rho\|w\|_{L^{2}}^{2}+\alpha_{1}\left\|\frac{\partial w}{\partial y}\right\|_{L^{2}}^{2}\right) \\
& \leq C\left(\rho\left\|w_{0}\right\|_{L^{2}}^{2}+\alpha_{1}\left\|\frac{\partial w_{0}}{\partial y}\right\|_{L^{2}}^{2}\right),
\end{aligned}
$$

where $C$ depends on $T$.

\section{G. Corollary 2}

If $w$ is a solution of Eqs (10) and (11) with $\phi=0$ then

$$
\begin{aligned}
& \sup _{0 \leq t \leq T}\left(\rho\left\|\frac{\partial w}{\partial y}\right\|_{L^{2}}^{2}+\alpha_{1}\left\|\frac{\partial^{2} w}{\partial y^{2}}\right\|_{L^{2}}^{2}\right) \\
& \leq C\left(\rho\left\|\frac{\partial w_{0}}{\partial y}\right\|_{L^{2}}^{2}+\alpha_{1}\left\|\frac{\partial^{2} w_{0}}{\partial y^{2}}\right\|_{L^{2}}^{2}\right),
\end{aligned}
$$

$C$ depends on $T$.

For this case Theorem is also satisfied.

\section{ACKNOWLEDGMENT}

The first author would like to express sincere gratitude to Professor Pengcheng Niu for guidance, constant encouragement and providing an excellent research environment.

\section{REFERENCES}

[1] C. Wu, "Numarical solution for Stoke's first problem for a heated generalized second grade fluid with fractional derivative," Appl. Numerical Mathematics, vol. 59, 2009, pp. 2571-2583.

[2] M. Jamil, A. Rauf, C. Fetecau, and N. A Khan, "Helical flows of second grade fluid due to constantly accelerated shear stress," Comm. Nonlinear Sci. Numer. Simul., vol. 16, 2011, pp. 1959-1969.

[3] Y. Yao and Y. Liu, "Some unsteady flows of a second grade fluid overa plane wall," Nonlinear Analysis: Real Word Applications, vol. 11 2010, pp. 4442-4450.

[4] B. I. Olajuwon, "Convection heat and mass transfer in a hydromagnetic flow of a second grade fluid in the presence of thermal radiation and thermal diffusion," Int. Comm. Heat Mass Transfer, vol. 38, 2011, pp 377-382.

[5] M. Pakdemirli, T. Hayat, M. Yurusory, S. Abbasbandy, and S. Asghar, "Perturbation analysis of a modified second grade fluid over a porous plate," Nonlinear Analysis: Real World Applications, vol. 12, 2011, pp. 1774-1785.

[6] C. Xue and J. Nie, "Exact solutions of the Rayleigh-Stokes problem for a heated generalized second grade fluid in a porous half space," App. Math. Modelling, vol. 33, 2009, pp. 524-531.

[7] W. C. Tan and T. Masuoka, "Stokes second problem for a second grade fluid in a porous half space with heated boundary," Int. J. Nonlinear Mech., vol. 40, 2005, pp. 512-522.

[8] S. Asghar, T. Hayatm, and P. D. Ariel, "Unsteady Couette flows in a second grade fluid with material properties," Comm. Nonlinear Sci. Numer. Simulation, vol. 14, 2009, pp. 154-159.

[9] S. Nadeem, T. Hayat, S. Abbasbandy, and M. Ali, "Effects of partial slip on a fourth grade fluid with variable viscosity: An analytic solution," Nonlinear Analysis: Real World Applications, vol. 11, 2010 pp. 856-868.

[10] M. Hussain, T. Hayat, S. Asghar, and C. Fetecau, "Oscillatory flows of second grade fluid in a porous space," Nonlinear Analysis: Real World Applications, vol. 11, 2010, pp. 2403-2414.

[11] K. R. Rajagopal and P. N. Kaloni, "Some remarks on boundary conditions for flows of fluids of the differential type," Cont. Mech. and its Applications, New York: Hemisphere Press, 1989, pp. 935-942.

[12] T. Hayat, S. Asghar, and A. M. Siddiqui, "Periodic unsteady flows of a non-Newtonian fluid," Acta Mech., vol. 131, 1998, pp. 169-175.

[13] A. M. Siddiqui, T. Hayat, and S. Asghar, "Periodic flows of a non-Newtonian fluid between two parallel plates," Int. J. Non-Linear Mech., vol. 34, 1999, pp. 895-899.

[14] T. Hayat, S. Asghar, and A. M. Siddiqui, "On the moment of a plane disk in a non-Newtonian fluid," Acta Mech., vol. 136, 1999, pp 125-131.

[15] T. Hayat, S. Asghar, and A. M. Siddiqui, "Some non steady flows of a non-Newtonian fluid," Int. J. Engng. Sci., vol. 38, 2000, pp. 337-346.

[16] J. Leray, "Etude de divers equations integrales nonlinearies et de quelques problems que posent lhydrodinamique," J. Math. Pures. Appl., vol. 12, 1933, pp. 1-82. 
[17] E. Hopf, "Uber die Anfangwertaufgaben fur die hydromischen Grundgleichungen," Math. Nach., vol. 4, 1951, pp. 213-321.

[18] J. Serrin, "On the interior regularity of weak solutions of the Navier-Stokes equations," Arch. Rational Mech. Anal., vol. 9, 1962, pp. 187-195.

[19] V. Scheffer, "Partial regularity of solutions to the Navier-Stokes equations," Pacific J. Math., vol. 66, 1976, pp. 535-552.

[20] L. Caffarelli, R. Kohn, and L. Nirenberg, "Partial regularity of suitable weak solutions of the Navier-Stokes equations," Comm. Pure Math., vol. 35,1982 , pp. $771-831$

[21] G. Tian and Z. Xin, "Gradient Estimation of Navier-Stokes equations," Comm. Anal. Geo., vol. 7, 1999, pp. 221-257.

[22] H. Sohr, "A regularity class for the Navier-Stokes equations in Lorentz spaces," J. Evolution Equations, vol. 1, 2001, pp. 441-467.

[23] H. Sohr, "A generalization of Serrin's regularity criterion for the Navier-Stokes equations," Quaderni Di Math., 2002.

[24] W. V. Wahl, "Regularity of weak solutions of the Navier-Stokes equation," in Proc. the 1983 Summer Institute.

[25] Y. Giga, "Solutions for semilinear parabolic equations in $L^{p}$ and regularity of weak solutions of the Navier-Stokes system," J. Differential equations, vol. 62, 1986, pp. 771-831.

[26] M. Struwe, "On partial regularity results for the Navier-Stokes equations," Comm. Pure Appl. Math., vol. 41, 1988, pp. 437-458.

[27] H. Kozono and H. Sohr, "Regularity criterion on weak solutions to the Navier-Stokes equations," Adv. Differential equation, vol. 2, 1997, pp. 535-554.

[28] C. Cao and J. Wu, "Global regularity for the 2D MHD equations with mixed partial dissipation and magnetic diffusion," Adv. in Math., vol. 226, 2011, pp. 1803-1822.

[29] R. Danchin and M. Paicu, "Global existence results for the anisotropic Boussinesq system in dimension two," arXiv:0809.4984v1 [math.AP], 19 September 2008.

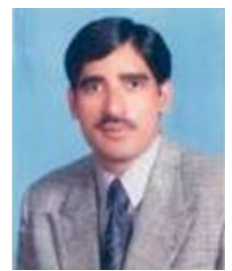

Tasawar Hayat did his Ph.D. in applied mathematics from Quaid-i-Azam university, Islamabad, Pakistan in 1999. Since then he has been teaching and research at Quaid-i-Azam university, Islamabad, Pakistan. He joined as lecturer in 1998 and has been promoted to Professor. Besides that he has been appointed as distinguished national professor by the Higher Commission of Pakistan. He has published extensively in Newtonian and non-Newtonian fluid mechanics. He has received a number of national and international awards, and is well known internationally.

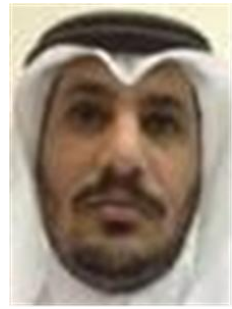

Hamed H. Alsulami completed his Ph. D. in 2005 from Arizona State university, Tempe, Arizona, USA. His research interest includes analysis, $C^{*}$-algebra, Banach algebra and differential equation. He has punlished papers recently in very good journals. He is a reviewer of several journals. He is one of the productive Scientist of the King Abdulaziz university, Sauid-i-Arabia. He completed several research projects.

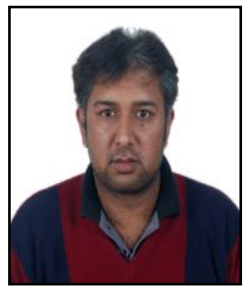

Saeed Rahman did his master degree in applied mathematics from Quaid-i-Azam university, Islamabad, Pakistan in 2005 and M.Phil in applied mathematics from Quaid-i-Azam university, Islamabad, Pakistan in 2007. From 2007-2011, he joined as a lecturer in COMSATS university, Pakistan. Now he is a PhD student in NWPU, China. His main research interest is partial differential equations. 